\title{
The occurrence of spring forms in tetraploid Timopheevi wheat is associated with variation in the first intron of the VRN-A1 gene
}

\author{
Andrey Borisovich Shcherban ${ }^{1 *}$, Aleksandra Aleksandrovna Schichkina ${ }^{2}$ and Elena Artemovna Salina ${ }^{1}$
}

From The International Conference on Bioinformatics of Genome Regulation and StructurelSystems Biology (BGRSISB-2016) Novosibirsk, Russia. 29 August-2 September 2016

\begin{abstract}
Background: Triticum araraticum and Triticum timopheevii are tetraploid species of the Timopheevi group. The former includes both winter and spring forms with a predominance of winter forms, whereas T. timopheevii is considered a spring species. In order to clarify the origin of the spring growth habit in T. timopheevii, allelic variability of the VRN-1 gene was investigated in a set of accessions of both tetraploid species, together with the diploid species Ae. speltoides, presumed donor of the $\mathrm{G}$ genome to these tetraploids.

Results: The promoter region of the VRN-A1 locus in all studied tetraploid accessions of both T. araraticum and T. timopheevii represents the previously described allele VRN-A1f with a 50 bp deletion near the start codon. Three additional alleles were identified namely, VRN-Alf-del, VRN-A7f-ins and VRN-Alf-del/ins, which contained large mutations in the first $\left(1^{\text {st }}\right)$ intron of VRN-A1. The first allele, carrying a deletion of $2.7 \mathrm{~kb}$ in a central part of intron 1, occurred in a few accessions of T. araraticum and no accessions of T. timopheevii. The VRN-Alf-ins allele, containing the insertion of a $0.4 \mathrm{~kb}$ MITE element about $0.4 \mathrm{~kb}$ upstream from the start of intron 1, and allele VRN-Alf-del/ins having this insertion coupled with a deletion of $2.7 \mathrm{~kb}$ are characteristic only for T. timopheevii. Allelic variation at the VRN-G1 locus includes the previously described allele VRN-G1a (with the insertion of a $0.2 \mathrm{~kb}$ MITE in the promoter) found in a few accessions of both tetraploid species. We showed that alleles VRN-Alf-del and VRN-GIa have no association with the spring growth habit, while in all accessions of T. timopheevii this habit was associated with the dominant VRN-Alf-ins and VRN-A1f-del/ins alleles. None of the Ae. speltoides accessions included in this study had changes in the promoter or $1^{\text {st }}$ intron regions of VRN-1 which might confer a spring growth habit. The VRN-1 promoter sequences analyzed herein and downloaded from databases have been used to construct a phylogram to assess the time of divergence of Ae. speltoides in relation to other wheat species.

(Continued on next page)
\end{abstract}

\footnotetext{
* Correspondence: atos@bionet.nsc.ru

${ }^{1}$ The Federal Research Center "Institute of Cytology and Genetics of Siberian Branch of the Russian Academy of Sciences", Lavrentiev ave. 10, Novosibirsk 630090, Russia

Full list of author information is available at the end of the article
} 
(Continued from previous page)

Conclusions: Among accessions of T. araraticum, the preferentially winter predecessor of $T$. timopheevii, two large mutations were found in both VRN-A1 and VRN-G1 loci (VRN-Alf-del and VRN-G1a) that were found to have no effect on vernalization requirements. Spring tetraploid T. timopheevii had one VRN-1 allele in common for two species (VRN-G1a), and two that were specific (VRN-Alf-ins, VRN-Alf-del/ins). The latter alleles include mutations in the $1^{\text {st }}$ intron of VRN-A1 and also share a $0.4 \mathrm{~kb}$ MITE insertion near the start of intron 1. We suggested that this insertion resulted in a spring growth habit in a progenitor of $T$. timopheevii which has probably been selected during subsequent domestication. The phylogram constructed on the basis of the VRN-1 promoter sequences confirmed the early divergence ( 3.5 MYA) of the ancestor(s) of the B/G genomes from Ae. speltoides.

Keywords: Allelic variation, Vernalization, VRN-1 gene, Promoter, First intron, Triticum, Aegilops

\section{Background}

Bread or common wheat, Triticum aestivum L., is one of the most important crops, providing a staple food for almost half of the human population world-wide. It is an allohexaploid (BBAADD genome, $2 \mathrm{n}=42$ ) that arose through hybridization of tetraploid wheat species (BBAA) with the diploid donor of D- genome. This event occurred about 8000 years ago $[1,2]$. Among tetraploid predecessors of common wheat are the emmer wheat $T$. dicoccoides (BBAA, $2 \mathrm{n}=28$ ), a wild progenitor from which modern tetraploid and hexaploid cultivated wheats were derived, and a separate group of species which belong to the section Timopheevi A. Filat. et Dorof. (GGAA).

Tetraploid Timopheevi wheats include the closely related species $T$. araraticum Jakubz. and T. timopheevii (Zhuk) Zhuk. T. araraticum grows primarily in Armenia, Azerbaijan, Georgia, Iran, Iraq and Turkey [3, 4] and originated from natural hybridization between $T$. urartu (AA, $2 \mathrm{n}=14$ ) and Ae. speltoides (SS, $2 \mathrm{n}=14$ ), the proposed donors of A- and G- genomes, respectively [1, 5-7]. $T$. araraticum is the ancestor of the domestic T. timopheevii. The latter has a restricted area of origin near Zanduri village in western Georgia [8]. Due to this feature, T. timopheevii is characterized not only by morphological homogeneity but also by a low cytogenetic variability [9].

The vernalization requirement, or necessity of cold treatment for the induction of flowering, is one of the most important traits affecting yield and adaptability of wheat crops. This characteristic has been intensively investigated in common wheat $T$. aestivum in which it is mainly controlled by three homoeologous $V R N-1$ loci: $V R N-A 1, V R N-B 1$ and $V R N-D 1$ [10-12]. Winter varieties are homozygous for the recessive alleles at the three $V R N-1$ loci, whereas spring wheat has dominant alleles at one or more of these loci. The dominant VRN-1 alleles are usually associated with mutations in two main regulatory regions of the $V R N-1$ gene, the promoter and first $\left(1^{\text {st }}\right)$ intron. Such mutations, namely, promoter deletions and insertions, and large deletions in intron 1, were initially detected in diploid T. monococcum (AA,
$2 \mathrm{n}=14$ ), common wheat $T$. aestivum and tetraploid $T$. durum $[10,13,14]$.

Recently, we characterized $V R N-1$ allelic diversity in a wide set of accessions representing wild tetraploid $T$. dicoccoides and three diploid A genome progenitor species [15]. The data showed that a set of dominant VRN1 alleles characteristic of wheat polyploids probably arose after allopolyploidization and were selected during domestication. Tetraploid Timopheevi wheats have not been systematically examined in this respect. One of the most conspicuous differences between $T$. araraticum and T. timopheevii is that the former includes both winter and spring forms with predominance of winter forms, whereas $T$. timopheevii is considered a spring species $[4,16]$. Hence, T. timopheevii represents a good model to study the origin of the spring growth habit in early wheat polyploids, a habit which may be primarily attributed to variation in the $V R N-1$ gene.

In order to identify and characterize which alleles of $V R N-1$ may affect the vernalization requirement in $T$. timopheevii, in this study we performed a comparative molecular analysis of the main regulatory regions of $V R N-1$ in Timopheevi wheat species. We also studied variability within $V R N-1$ in the diploid Ae. speltoides, the proposed donor of $\mathrm{B} / \mathrm{G}$ - genome.

\section{Results \\ $V R N-1$ allelic variability in tetraploid Timopheevi wheat Promoter region}

Vernalization sensitivity in tetraploid wheat $T$. araraticum and T. timopheevii is controlled by alleles at the two homoeologous loci, VRN-A1 and VRN-G1. The specific primers Vrn1AF and Int1R were used to identify variation in the length of the promoter region of the VRN-A1 locus, as described by Yan et al. [13] (Table 1; Fig. 1). All studied accessions of both tetraploid species yielded a PCR product of approximately $0.65 \mathrm{~kb}$ (Additional file 1; Fig. 2a). We sequenced the PCR products obtained with VrnA1F/ Int1R primers for a selected set of accessions representing these species (Table 2). 
Table 1 PCR markers for determining the presence of different alleles of VRN-A1, VRN-G1 in tetraploid Timopheevi wheats and diploid Ae. speltoides

\begin{tabular}{|c|c|c|c|c|c|c|}
\hline PCR marker & Name & Primer $\left(5^{\prime} \rightarrow 3^{\prime}\right)$ & Target allele(s) & $\begin{array}{l}\text { Expected pro-duct } \\
\text { size (bp) }\end{array}$ & $\begin{array}{l}\text { Annealing } \\
\text { temp. }\left({ }^{\circ} \mathrm{C}\right)\end{array}$ & Reference. \\
\hline \multirow[t]{4}{*}{ VRN-A1 marker ${ }^{\mathrm{a}}$} & Vrn1AF & GAAAGGAAAAATTCTGCTCG & VRN-A1f & 655 & 55 & [13] \\
\hline & $\operatorname{lnt1R}$ & GCAGGAAATCGAAATCGAAG & VRN-Aif-del & & & \\
\hline & & & VRN-A1f-ins & & & \\
\hline & & & VRN-Alf-del/ins & & & \\
\hline \multirow[t]{2}{*}{ VRN-A1 Non-deletion } & $\ln \operatorname{tr} 1 / C / F$ & GCACTCCTAACCCACTAACC & VRN-A1f & 1068 & 56 & [14] \\
\hline & Intr1/AB/R & TCATCCATCATCAAGGCAAA & VRN-Alf-ins & & & \\
\hline \multirow[t]{5}{*}{ VRN-A1 insertion } & Intr 1 & ATCATCTTCTCCACCAAGGG & VRN-A1f & 1480 & 50 & [15] \\
\hline & Intr1insR & AATGAACAGCACGGAAACAG & VRN-Alf-ins & 1910 & & \\
\hline & & & VRN-A1f-del/ins & & & \\
\hline & mitef & CAGGTAAGGTATGAGGTGAC & VRN-Alf-ins & 370 & 52 & - \\
\hline & miter & GATTCCAAATGAGAAGATGAGG & VRN-A1f-del/ins & & & \\
\hline \multirow[t]{4}{*}{ VRN-A1 deletion of $2.7 \mathrm{~kb}$} & Intr1/A/F2 & AGCCTCCACGGTTGAAAGTAA & VRN-Aif-del & $\sim 2500$ & 56 & [14] \\
\hline & Intr4 & GCGCCATTAGGGAGGCACTT & VRN-A1f-del/ins & & & \\
\hline & delf & ACATGTAAGCAGATCCTATCGA & VRN-Aif-del & 450 & 53 & - \\
\hline & delr & TGCTTTAGATCTTTCTTCACGG & VRN-A1f-del/ins & & & \\
\hline \multicolumn{7}{|l|}{ VRN-G1 marker ${ }^{\mathrm{a}}$} \\
\hline for T. araraticum/ & P1 & TACCCCTGCTACCAGTGCCT & VRN-G1 & 900 & 55 & [23] \\
\hline T. timopheevii & P5 & GGCCAACCCTACACCCCAAG & VRN-GIa & 1100 & & \\
\hline \multirow[t]{2}{*}{ for Ae. speltoides } & P8 & CTAGGACTGGCGAGTATCTT & VRN-1 & 900 & 55 & - \\
\hline & P10 & GAGAACCGGGCCAACCCTAC & & & & \\
\hline \multicolumn{7}{|l|}{ VRN-G1 Non-deletion } \\
\hline for T. araraticum/ & $\mathrm{Ex} 1 / \mathrm{C} / \mathrm{F}$ & GTTCTCCACCGAGTCATGGT & VRN-G1 & 1530 & 56 & [14] \\
\hline T. timopheevii & Intr1/B/R4 & CAAATGAAAAGGAATGAGAGCA & VRN-GIa & & & \\
\hline \multirow[t]{2}{*}{ for Ae. speltoides } & $\mathrm{Ex} 1 / \mathrm{C} / \mathrm{F}$ & GTTCTCCACCGAGTCATGGT & VRN-1 & 1530 & 60 & [14] \\
\hline & Intr1/D/R4 & AAATGAAAAGGAACGAGAGCG & & & & \\
\hline
\end{tabular}

${ }^{a}$ These diagnostic markers detect allelic variation at the promoter regions. In other cases variation within intron 1 of corresponding genes is detected

The promoter sequences of all studied accessions were $99 \%$ homologous to each other and to the previously published VRN-A1f promoters from T. timopheevii and T. araraticum (GQ451751-451753 and GQ451762-451765, respectively). Compared to the other known VRN-A1 sequences, the $V R N-A 1 f$ promoter has a $50 \mathrm{bp}$ deletion in the position $-63 \mathrm{bp}$ from the start codon (Fig. 1). A few indels were also identified between the studied sequences, including a 1 bp deletion ( $\mathrm{C}$ nucleotide within $\mathrm{C}$-rich segment upstream from the CArG-box) in 4 out of 8 accessions of T. araraticum and four nucleotide substitutions. The four studied VRN-A1 promoter sequences of $T$. timopheevii have no specific indels compared to the respective sequences of $T$. araraticum.

For analysis of the VRN-G1 locus, the primers P1/P5 were used to amplify an approximately $0.9 \mathrm{~kb}$ region of the promoter sequence (Table 1; Fig. 1). 41 accessions of $T$. araraticum and 3 accessions of T. timopheevii gave the expected PCR product, while 4 accessions of $T$. araraticum and 1 accession of T. timopheevii generated a larger PCR product of about $1.1 \mathrm{~kb}$ (Fig. 2b). Sequencing of the product from one of the former accessions (PI 427403) showed that it is highly homologous with the VRN-G1 promoter from T. araraticum (KR055682), while those from accessions PI 654340 and PI 119442,- representatives of the latter group, are almost identical with the previously published $V R N-G 1$ a promoter from T. timopheevii (GQ451755) (Table 2). The last promoter contains a MITE (miniature inverted-repeat transposable element)like insertion located $99 \mathrm{bp}$ upstream from the stop codon (Fig. 1). The MITE consisted of 215 bp, including a 9 bp host duplication (CTCCGCCCC) and 29 bp inverted repeats at the ends (Fig. 3). The sequence was found to be $92 \%$ identical to a foldback element inserted in the VRNA1a allele of T. aestivum (AY616458). Thus, the recessive $V R N-G 1$ allele is characteristic of most $T$. araraticum and T. timopheevii accessions, whereas the VRN-G1a allele occurs with less frequency in both species. 


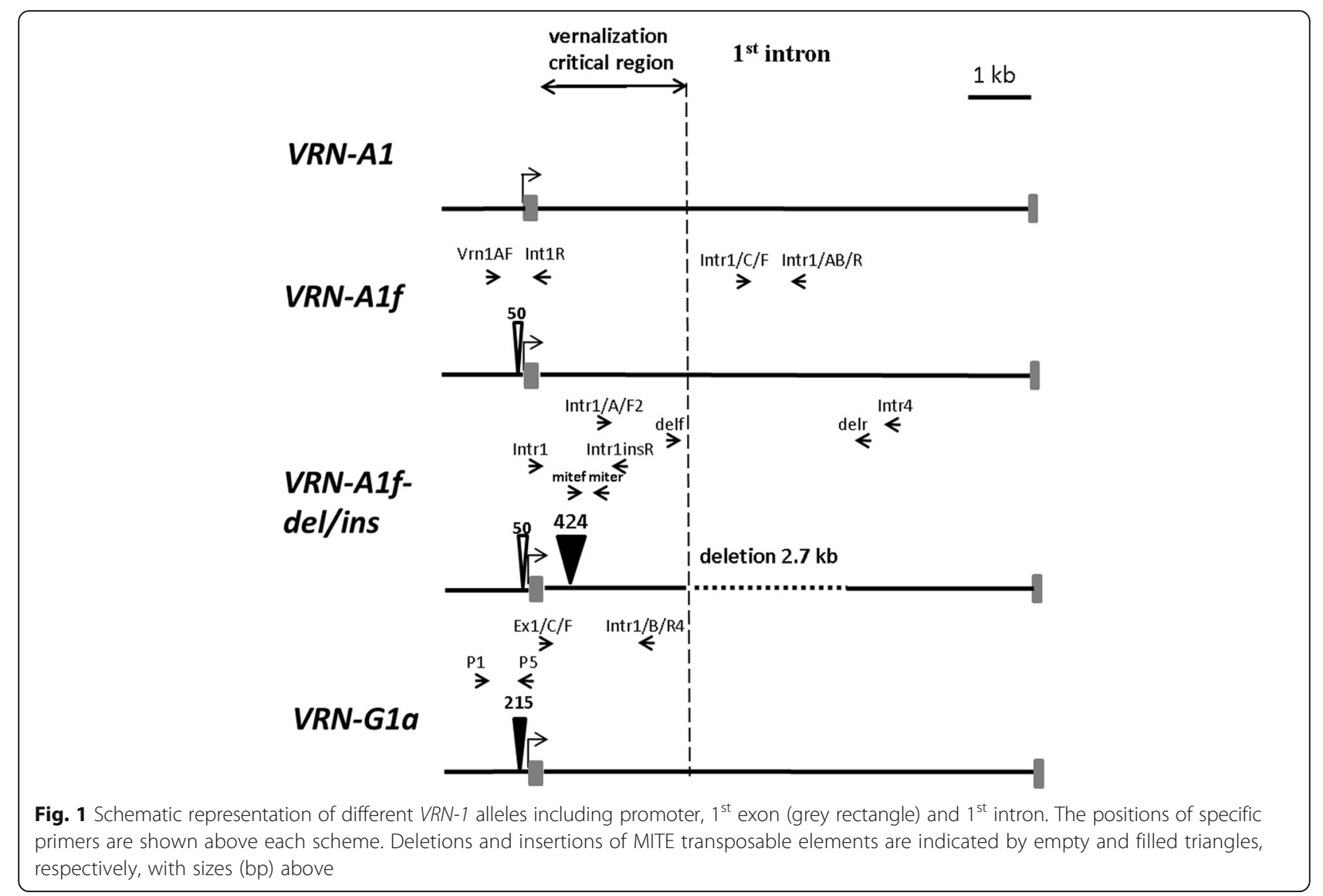

\section{$7^{\text {st }}$ intron}

The primer pair Intr $1 / C / F$ and $\operatorname{Intr} 1 / A B / R$ for the $1^{\text {st }}$ intron of VRN-A1 (Table 1; Fig. 1) were previously used to confirm the presence of the recessive allele of $T$. aestivum with no significant changes (deletions or insertions) in intron 1 [14]. 41 accessions of T. araraticum (including 4 accessions represented in Fig. 2) and one accession of T. timopheevii yielded a PCR product of approximately $1 \mathrm{~kb}$, indicating the presence of the intact $1^{\text {st }}$ intron within the $V R N-A 1$ gene (Fig. 2c). The remaining accessions of both species gave no PCR products with this pair of primers. To identify the possible changes explaining this last pattern, the $1^{\text {st }}$ intron region of $V R N-A 1$ was split into subregions which were amplified separately by different combinations of primers.

The central portion of intron 1 flanked by the primers Intr1/A/F2 and Intr4 (Table 1; Fig. 1) can hardly be amplified in the case of the recessive $V R N-A 1$ allele due to its large size $(\sim 5 \mathrm{~kb})$. However, for four $T$. araraticum and three T. timopheevii accessions a PCR product of approximately $2.5 \mathrm{~kb}$ was amplified using these primers (data not presented). This indicated the presence of a deletion preventing amplification with pair Intr1/C/F // Intr1/AB/R (Fig. 1). The one-sided sequencing of the product allowed us to establish the boundaries of the deletion and its size- $2.7 \mathrm{~kb}$ by comparison with the known recessive $V R N-A 1$ alleles. The primers delf and delr were designed bordering the deletion and giving a 450 bp fragment (Table 1; Fig. 2d). Hence, the deletion of $2.7 \mathrm{~kb}$ in intron 1 of $V R N-A 1$ is characteristic of three studied accessions of T. timopheevii and 4 out of 45 accessions of $T$. araraticum (Table 2).

Using the primer combination Intr1// Intr1insR, all $T$. timopheevii accessions yielded a larger PCR product compared with accessions of $T$. araraticum (data not shown). The sequencing of this product showed the presence of an insertion located $435 \mathrm{bp}$ downstream from the end of exon 1 (Fig. 3). This insertion of $424 \mathrm{bp}$ long was found to be $99 \%$ identical to the previously identified MITE element in the VRN-A1f-like allele of $T$. militinae [17]. In order to confirm this result, we used the primer pair mitef/ miter targeting the right junction of the insertion (Table 1; Fig. 1). All accessions of $T$. timopheevii yielded a product of $370 \mathrm{bp}$, whereas no product was obtained with the $T$. araraticum accessions (Fig. 2e). Therefore, unlike the $2.7 \mathrm{~kb}$ deletion, the $0.4 \mathrm{~kb}$ MITE insertion in intron 1 is a specific marker for the $V R N-A 1$ allele of $T$. timopheevii.

Here, we designated the VRN-Alf alleles with different mutations in the $1^{\text {st }}$ intron as VRN-A1f-del, VRN-Alf-ins 


\section{GGAA}

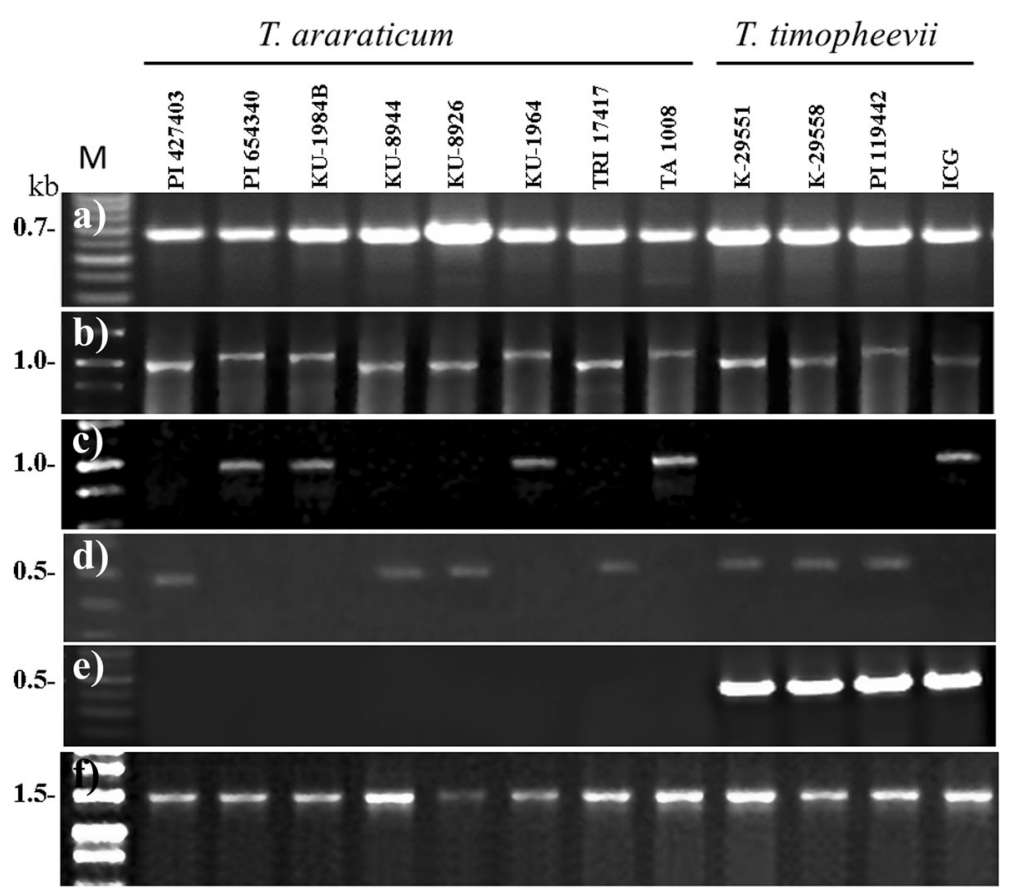

Fig. 2 PCR amplification with primers VrnA1F/Int1R (a), P1/P5 (b), Intr1/C/F // Intr1/AB/R (c), delf/delr (d), mitef/miter (e), Ex1/C/F // Intr1/B/R4 (f). Accession numbers, species and genotype are given at the top

and VRN-A1f-del/ins (the $2.7 \mathrm{~kb}$ deletion, the $0.4 \mathrm{~kb}$ insertion and both mutations, respectively) (Table 2).

The primer pair Ex1/C/F and Intr1/B/R4 was designed as a positive control for the absence of large mutations in intron 1 of the $V R N-B 1 / G 1$ locus [14] (Table 1). Using these primers, all accessions of $T$. araraticum and $T$. timopheevii yielded a $1530 \mathrm{bp}$ PCR product indicating an intact $1^{\text {st }}$ intron region (Fig. 2f).

Thus, the tetraploid wheats $T$. araraticum and T. timopheevii displayed most structural variation at the $1^{\text {st }}$ intron region of $V R N-A 1$, including the $0.4 \mathrm{~kb}$ MITE insertion specific for T. timopheevii. Unlike $V R N-A 1$, the $V R N-G 1$ locus showed no variation in the $1^{\text {st }}$ intron, while in the promoter a restricted variation was revealed (allele VRN-G1a) in some accessions of both species.

\section{Evaluation of growth habit in tetraploid accessions}

To assess the impact of $V R N-1$ alleles on vernalization requirements in tetraploid wheat, we selected the accessions of T. araraticum and T. timopheevii presented in Table 2. Notably, in all the studied accessions of T. araraticum the alleles VRN-A1f, VRN-A1f-del and VRN-G1a were associated with a winter growth habit, implying that these alleles have no effect on vernalization requirements. All four accessions of T. timopheevii with alleles $V R N-A 1 f$-del/ins (insertion + deletion in intron 1) and $V R N$-Alf-ins (insertion in intron 1, no deletion) were spring types. Consequently, the insertion of the MITE element in the $1^{\text {st }}$ intron of $V R N-A 1$ locus is probably associated with the spring growth habit of T. timopheevii.

\section{VRN-1 allelic variation in Ae. speltoides Promoter region}

The primer pair P1 and P5 successfully used for amplification of the VRN-G1 promoter in T. araraticum and T. timopheevii (see above) gave no PCR products in the case of Ae. speltoides. Primers pair P8/P10 was designed, so that primer P8 anneals close to the 3 '-end of the annealing site for P1 primer, while P10 overlaps the 3'half of the annealing site for P5 (Table 1). This pair yielded an expected product of approximately $0.9 \mathrm{~kb}$ in all the studied accessions of Ae. speltoides (data not presented). This indicates the presence of the intact VRN-G1 promoter, except under circumstances where flanking sequences may have been modified, preventing amplification with P1/P5. To further analyze allelic variation in the VRN-G1 promoter, we randomly selected 7 accessions of Ae. speltoides and sequenced the corresponding PCR products obtained with P8/P10 primers.

All 7 sequences were highly similar to each other ( $\geq 98 \%$ homology). Minor variation between individual sequences was found, including nucleotide substitutions and small deletions of up to $4 \mathrm{bp}$. Most of this variation was upstream from the conserved region encompassing 


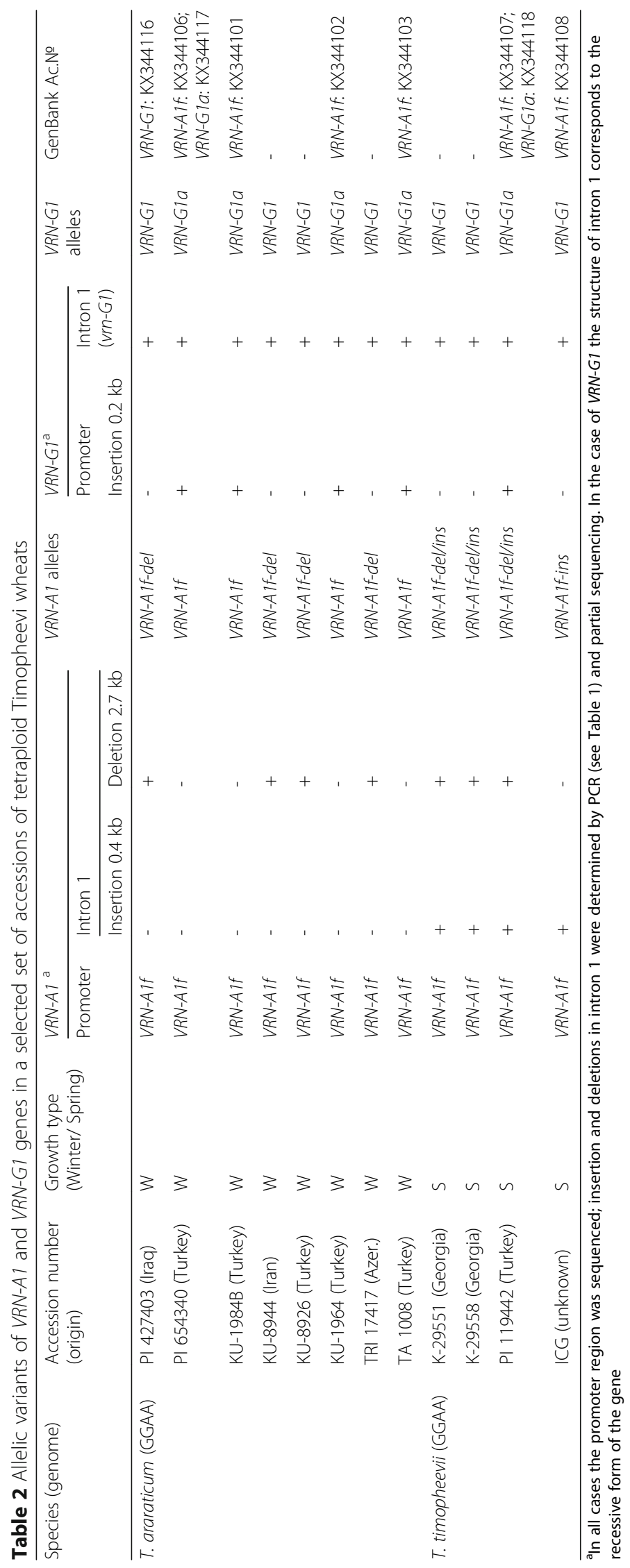




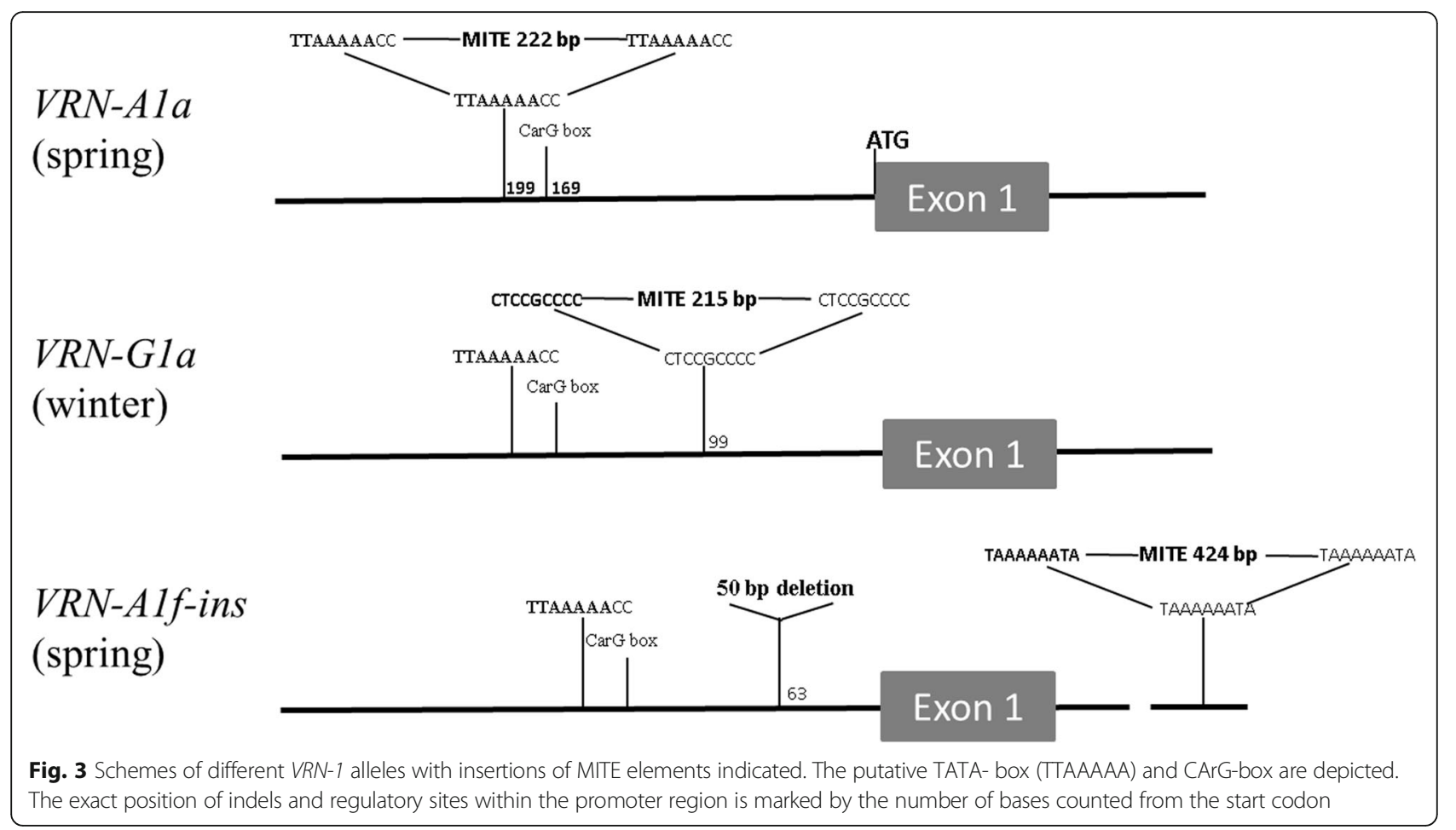

about $0.3 \mathrm{~kb}$ from the start codon and containing the putative regulatory sites $[10,13,18]$. A conspicuous polymorphism was found in the tandem track of AGCC repeats $\sim 0.6 \mathrm{~kb}$ upstream from the start codon. All known $V R N-B 1 / G 1$ sequences of wheat polyploids contain 4 repeats, while in the studied sequences of Ae. speltoides the number of repeats varies from two (K-2278, I-551352, I570060) up to three (K-453, K-3257, K-911, K-443).

The VRN-G1 promoter sequences of Ae. speltoides displayed a similar homology (92-93 \%) to the corresponding sequences of wild tetraploid species $T$. araraticum and $T$. dicoccoides accessed from databases. Sequence variation as compared with tetraploids included a few small deletions and insertions up to $7 \mathrm{bp}$, the largest deletion of $21 \mathrm{bp}$ located about $0.5 \mathrm{~kb}$ upstream from the start codon is characteristic of all studied Ae. speltoides sequences.

\section{$1^{\text {st }}$ intron}

All Ae. speltoides accessions gave no the expected PCR product of $1.5 \mathrm{~kb}$ using primers Ex1/C/F and Intr1/B/R4 which anneal with exon 1 and intron 1 sequences, respectively. Taking into account the relationship between Ae. speltoides and Ae. tauschii (DD genome), instead of Intr1/B/R4 we tested the Intr1/D/R4 primer pair having 2 mismatches specific for Ae. tauschii in the same annealing site (Table 1). The presence of the expected PCR product in the latter case (data not shown) indicates a divergence within the Intr1/B/R4 annealing site as the cause of the negative result using the first primer pair.

\section{Phylogenetic analysis of $V R N-1$ promoter sequences}

To assess the degree of divergence in $V R N-1$ promoter sequences between the diploid species that donated their genomes to polyploids, and the different polyploid wheats (tetra-, or hexaploid-), we constructed a neighbor-joining phylogram based on the alignment of the nucleotide sequences obtained in this research and downloaded from databases (see Methods). In general, three main groups form the distinct branches on the tree (Fig. 4). The sequences of Ae. speltoides originated from the same branch as those of the B/G-genomes of polyploids, but diverged comparatively early about 3.6 MYA according to our estimation. This date is comparable with the date of divergence of the D-genome group (3.2 MYA) which occupies an intermediate position between $\mathrm{B} / \mathrm{G}$ and A- groups. The latter falls into a separate branch of the diploid T. monococcum and subgroup comprising the diploid $T$. urartu and A-genome sequences of polyploids. Within this subgroup the previously established date of divergence of the allopolyploid branch (0.65 MYA) was used for calibration (see Methods).

\section{Discussion}

High plasticity and adaptability of polyploid wheat to growth at various latitudes is typically provided by allelic variation of genes which control sensitivity to vernalization and photoperiod (VRN and $P P D$, respectively). Following extensive molecular genetic studies of cereal plants, the integrated model of flowering regulation including both factors was proposed [19]. According to this model, the 


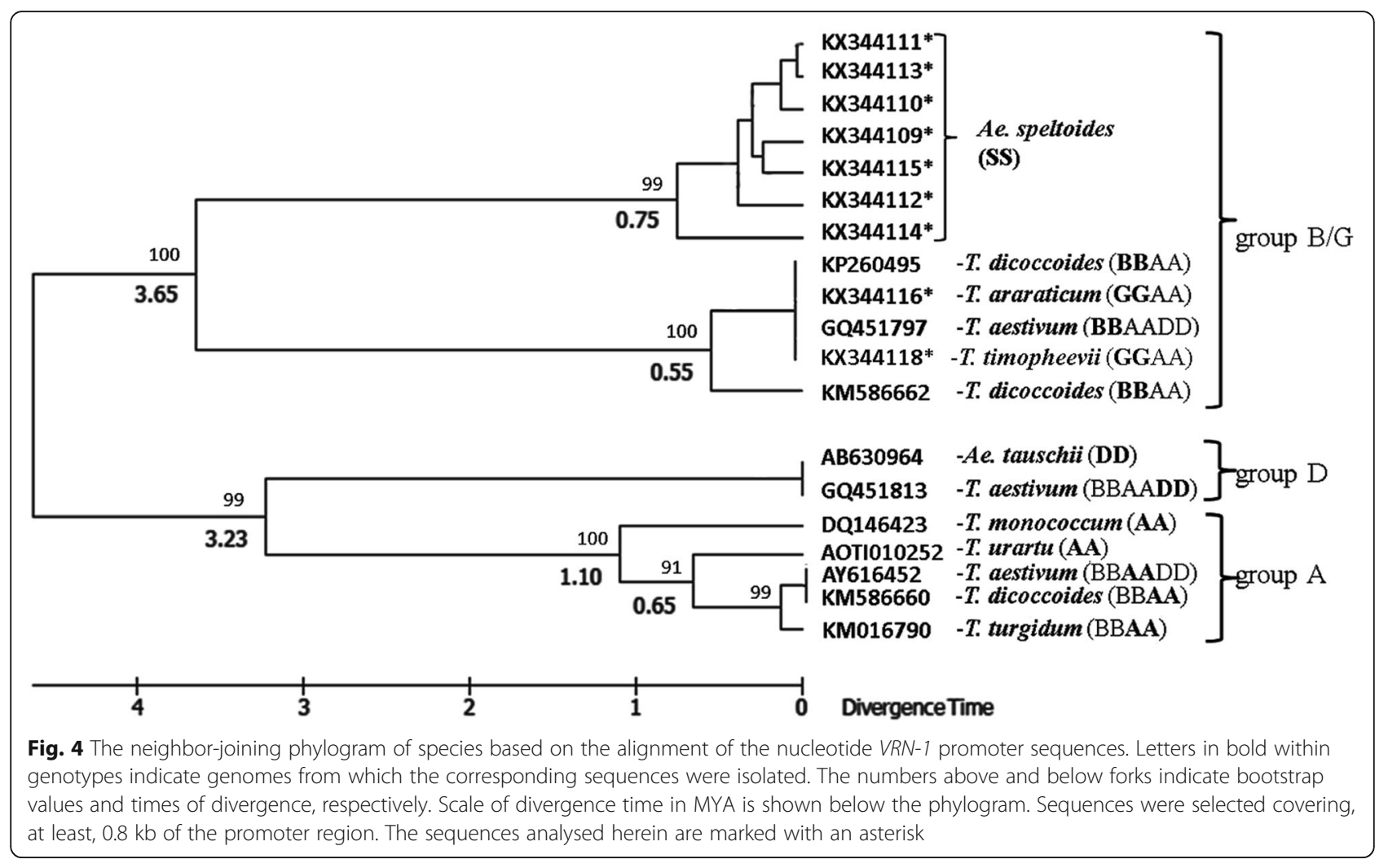

$V R N-1$ gene plays a key role in combining different signal transduction pathways via two main regulatory regions in its structure, promoter and $1^{\text {st }}$ intron. Mutations in these regions have an impact on $V R N-1$ expression and enables plants to significantly modulate vernalization requirements and flowering time [20-24]. However, the specific mechanisms by which each region exerts their effects are still to be established.

\section{The origin of the spring growth habit in tetraploid Timopheevi wheat}

Most of the wild Triticeae species, both diploid and polyploid, have a winter growth habit, suggesting that the recessive $V R N-1$ allele is the ancestral form. By contrast, there are many cultivated polyploid wheat varieties with a spring growth habit and with at least one dominant $V R N-1$ allele [25]. Polyploid wheat is divided into two evolutionary lineages, Emmer (BBAA) and Timopheevi (GGAA), which formed through hybridization between $T$. urartu and Ae. spetoides, and divergence of the first wild tetraploids $T$. diccocoides (BBAA) and $T$. araraticum (GGAA) occurred over the course of several tens of thousands years [26]. The appearance of spring forms in the Emmer group is presumably associated with mutations in the promoter or $1^{\text {st }}$ intron of $V R N-A 1$ locus, mainly, deletions of different lengths [15]. In order to study the origin of the spring growth habit in Timopheevi wheat, we compared the wild species $T$. araraticum to its domesticated spring descendant, T. timopheevii.

The promoter region of $V R N-A 1$ in both tetraploid species displays a high level of homogeneity and is almost identical to the previously described VRN-A1f allele of $T$. timopheevii [27]. The characteristic of this allele is a 50 bp deletion near the start codon (Fig. 1). Such a deletion has not been described in Emmer or diploid wheat species, so it is probably specific for Timopheevi wheat. The VRN-G1 locus both in the promoter and its $1^{\text {st }}$ intron showed no significant variation between the two tetraploid species. The single VRN-G1a allele found with a $0.2 \mathrm{~kb}$ insertion has a low frequency among $T$. araraticum accessions studied here $(8 \%)$ and was also found in two accessions of $T$. timopheevii, the previously studied GQ451755 and presented here KX344118 (Table 2). Evaluation of growth habits demonstrated that neither VRN-A1f nor VRN-G1a are responsible for spring type growth, since all accessions carrying these alleles were winter types. Simultaneously, we found an obvious species-specific polymorphism within the $1^{\text {st }}$ intron region of $V R N-A 1$. Alterations in this region include the $0.4 \mathrm{~kb}$ MITE insertion and the $2.7 \mathrm{~kb}$ deletion located about $0.4 \mathrm{~kb}$ and $\sim 3 \mathrm{~kb}$ downstream from the start of intron 1, respectively (Fig. 1). Recently, Ivanicova et al. [17] described the VRN-A1flike allele containing both mutations in lines of spring bread wheat with introgression of genetic material from 
T. militinae Zhuk. and Migush., the free-threshing mutant that was selected from a single specimen of $T$. timopheevii [28]. These lines were characterized by a significant difference in flowering time- the effect associated with the VRN-Alf-like allele. Here, we have demonstrated for the first time that the MITE insertion and the deletion in the $1^{\text {st }}$ intron of VRN-A1 are of independent origin and have different effects on the growth habit. The deletion of $2.7 \mathrm{~kb}$ obviously appeared before the divergence of $T$. timopheevii from $T$. araraticum, since 4 accessions of the last species contained this single mutation (allele VRN-A1f-del). In addition, we found the MITE insertion in no accessions of $T$. araraticum and all the studied accessions of T. timopheevii where it was combined with the $2.7 \mathrm{~kb}$ deletion (VRN-A1f-del/ ins) in 3 accessions, or present as a single mutation (VRN-A1f-ins) in 1 accession (Table 2). Therefore, we suggested that the spring growth habit arose in an ancestor of the modern T. timopheevii due to insertion of MITE within the $1^{\text {st }}$ intron of $V R N-A 1$ and this mutation has been selected during domestication of this species.

Previous data support the importance of intron 1 of $V R N-1$ gene in the vernalization response and control of heading time, and the proposed "vernalization critical" regulatory region has been located encompassing $\sim 3 \mathrm{~kb}$ from the start of intron 1 (Fig. 1) [11, 14]. A number of mutations (deletions, insertions etc.) in this region have been associated with a spring growth habit in different crops $[14,21,29,30]$. It was suggested that all these mutations may affect epigenetic chromatin states, resulting in a higher basal level of VRN-1 expression which is necessary for inducing a spring type of growth $[22,30]$. Previously, we described the dominant allele $V R N-B 1 c$ as having large alterations (deletion and duplication) within the 5'-flanking part of intron 1 and found that these mutations resulted in a higher level of $V R N-1$ transcription and subsequent reduction in flowering time compared to the allele without such mutations [23, 24]. Another example is the VRN-A1ins allele of the diploid $T$. monococcum (AA) with a $0.5 \mathrm{~kb}$ insertion in the same region which is associated with a spring growth habit in $30 \%$ accessions of this species $[15,21]$.

The host duplication of MITE in the first intron of $V R N-A 1$ resembles the TATA-box sequence (Fig. 3). A similar duplication is characteristic of a MITE inserted in the TATA-box of the dominant VRN-A1a allele of $T$. aestivum [13]. The last mutation may create an alternative promoter, or regulatory sites allowing modulation of transcription and, consequently, the vernalization requirement. Interestingly, the MITE insertion in VRNG1a has a completely different duplication despite a high homology with the previous element (Fig. 3). Therefore, mobile elements are capable of inducing different modes of genetic regulation depending on their structure and region of insertion.

\section{Allelic variation in the $V R N-1$ gene confirms the early divergence of $A e$. speltoides from the progenitor of the $B / G$ genomes}

The two wild tetraploid wheat species, $T$. dicoccoides and $T$. araraticum, are relatively similar in plant morphology, but they differ in their genomic constitution (BBAA and GGAA, respectively). The identity of the donor(s) of the $B$ and $G$ genomes remains open to speculation. Based on the analysis of different nuclear and chloroplast loci, it was concluded that the ancestor of these genomes was a member of section Sitopsis of the genus Aegilops - most likely, Ae. speltoides Tausch $[5,7,31]$. Recently, we studied allelic variation within the $V R N-B 1$ gene of $T$. dicoccoides and found a low level of polymorphism compared with $V R N-A 1$ [15]. The similar conservatism was shown here for the VRN-G1 locus of Timopheevi wheats, except the $V R N-G 1 a$ allele was found to have no effect on vernalization requirements. The question then arose whether the structural peculiarities of the both loci were inherited from Ae. speltoides, the putative progenitor of $\mathrm{B}$ and $\mathrm{G}$ genomes.

Our results showed that divergence of the $V R N-1$ locus in Ae. speltoides affected the annealing sites for primers usually used to target the corresponding $V R N$ $B 1 / G 1$ genes in polyploids. So, in the case of the promoter region, we used a newly designed pair of primers, while for the $1^{\text {st }}$ intron the $\mathrm{D}$ - genome specific pair was used (Table 1). In the both cases, PCR products were obtained corresponding to the recessive form of the $V R N-1$ gene.

The sequencing of the $V R N-1$ promoter region from 7 randomly selected accessions of Ae. speltoides showed almost no variation inside or near the probable regulatory sites, such as the TATA-box, VRN- and CArG-boxes etc., established earlier [10, 13, 18, 32, 33]. The majority of variation was found upstream of the regulatory sites, including a short tandem repeat AGCC track which consists of 2-3 repeat motifs in all the studied Ae. speltoides sequences, whereas in the known $V R N-A 1$, $V R N-D 1$ and $V R N-B 1 / G 1$ sequences it includes 2,3 and 4 repeats, respectively. Hence, there is a tendency toward an increasing number of repeats during evolution of the $\mathrm{B} / \mathrm{G}$ - genome. Whether this tendency is due to some regulatory effect on $V R N-B 1 / G 1$ locus, or a result of stochastic processes, like genetic drift, is as yet unknown.

The VRN-1 promoter sequences of Ae. speltoides were similarly homologous to the corresponding database sequences of wild tetraploids $T$. dicoccoides and $T$. araraticum with the maximum level of homology 93\% in both cases. Of note, the latter sequences were almost identical (99-100 \% homology) to those of hexaploid $T$. 
aestivum, implying a high conservation of $V R N-B 1$ promoter structure throughout both tetraploid and hexaploid stages of wheat evolution. In order to assess the relative rates of divergence between A-, B- and D- genomes of polyploids and corresponding diploid donors, we constructed a phylogenetic tree using the $V R N-1$ promoter sequences analysed herein and those available in the public databases (Fig. 4). Divergence time was estimated using the molecular clock approach (see Methods). Based on this estimation, the progenitor of B/G genomes diverged from Ae. speltoides much earlier ( 3.5 MYA), than the progenitor of A-genome did from T. urartu, closest to the diploid donor of A- genome, and slightly earlier than D- genome branch occurred ( 0.65 and 3.2 MYA, respectively). An intermediate position of the latter branch is consistent with the proposal of Marcussen et al. [2] that the ancestor of D-genome appeared due to a homoploid hybridization event between the progenitors of "A- and B- genome lineages". The times of divergence of the main branches are earlier in our model as compared with the previous one, but close to the dates presented by Gill et al. [34] in the scheme of wheat phylogeny. Therefore, the obtained results confirm previous data indicating a fairly distant position of Ae. speltoides from the ancestors of polyploid forms and show that the VRN-1 promoter region may serve as a good marker of phylogenetic relationships in the Triticeae tribe.

\section{Conclusions}

In the present study we investigated variability in the promoter and $1^{\text {st }}$ intron regions of the vernalization gene $V R N-1$ in different accessions of tetraploid species of Timopheevi group and their diploid G- genome progenitor, Ae. speltoides. Our results indicated that the occurrence of spring forms in tetraploid T. timopheevii is attributed to variation in the $1^{\text {st }}$ intron region of $V R N$ A1 gene, namely, the insertion of a MITE element within "vernalization critical" parts of this region. All the studied accessions of $T$. araraticum with the winter growth type have no mutations of this kind despite the presence of other changes affecting regulatory regions in both $V R N-A 1$ and VRN-G1 loci. This implies that the insertion of the MITE occurred in an ancestor of the modern T. timopheevii and has probably been selected during domestication of this species. The allelic variability of the VRN-1 locus in the diploid Ae. speltoides was as low as in the tetraploid descendants to which the former species donated the B and G-genomes. The VRN-1 promoter sequences were used to construct a phylogram which confirmed an early divergence of Ae. speltoides from the progenitor of the $\mathrm{B} / \mathrm{G}$ genomes (3.6 MYA). Topology of the tree and the dates of divergence from the main branches are in agreement with other data, making the promoter region of $V R N-1$ gene useful for targeting of different genomes and estimation of their phylogeny.

\section{Methods}

\section{Plant material and DNA extraction}

The plant material included tetraploid wheat species $T$. araraticum and T. timopheevii (45 and 4 accessions, respectively), and 23 accessions of Ae. speltoides. These accessions were selected from the different genebanks of Russia, Syria, Japan, and the USA (Additional file 1).

DNA was extracted from 7-day-old seedlings following [35]. Leaves from 3-5 seeds per accession were homogenised using a FastPrep-24 instrument (MP Biomedicals, USA).

\section{PCR}

PCR primers reported in $[13,14,23,24]$ were used to identify different alleles of VRN-A1 and VRN-G1 loci in the material studied (Table 1; Fig. 1). To further discriminate different indels in the $1^{\text {st }}$ intron region of the $V R N$ A1 locus we designed 2 pairs of primers: mitef/ miter and delf/ delr for detection of a $0.4 \mathrm{~kb}$ insertion and deletion of $2.7 \mathrm{~kb}$, respectively. In the case of Ae. speltoides we designed the primers $\mathrm{P} 8$ and P10, instead of previously used P1/P5 primers, to amplify the VRN-G1 promoter region covering $\sim 900 \mathrm{bp}$ upstream from the start codon (Table 1).

The PCR conditions were as described in [15]. Amplicons were separated through $1 \%$ agarose gel.

\section{Sequencing of PCR products}

Amplified DNA fragments were purified from an agarose gel using a QIAquick PCR purification kit (QIAGEN, Germany), then directly sequenced in both directions using an ABI PRISM Dye Terminator Cycle Sequencing ready reaction kit (Perkin Elmer Cetus, USA). Sequencing was conducted using resources of SB RAS Genomics Core Facilities (Novosibirsk, Russia, http://sequest.niboch.nsc.ru). GenBank accession numbers of $V R N-A 1$ and VRN-G1 sequences from tetraploid species (KX344100-08 and KX344116-18, respectively) are partially represented in Table 2. The VRN-1 promoter sequences of Ae. speltoides were deposited under Ac.№ KX344109-15.

\section{Sequence analysis}

Multiple sequence alignments and the subsequent phylogenetic analysis were carried out using the ClustalW program and MEGA4 software [36, 37]. A phylogenetic tree was constructed using the Neighbor-Joining algorithm and 500 bootstrap replicates. Divergence time was assessed by MEGA4 using calibration against a previously established date of formation of the allotetraploid wheat of about 0.5-0.6 Million Years Ago (MYA) [1, 38]. 


\section{Evaluation of growth habit}

Growth habits of the diploid and tetraploid accessions containing different $V R N-1$ alleles were determined as described in [15] from two replicates in 2015-2016 (Table 2).

\section{Additional file}

Additional file 1: Plant material used in the analysis, its origin and VRN-1 genotype. (DOCX $26 \mathrm{~kb}$ )

\section{Acknowledgements}

We would like to thank Dr. O. P. Mitrofanova for supplying seeds of wheat species. We are grateful to the Genomics Core Facility at the Institute of Chemical Biology and Fundamental Medicine SB RAS (Novosibirsk, Russia) for sequencing of PCR products.

\section{Declarations}

This article has been published as part of BMC Plant Biology Volume 16 Supplement 3, 2016: Selected articles from BGRS\SB-2016: plant biology. The full contents of the supplement are available online at http://bmcplantbiol. biomedcentral.com/articles/supplements/volume-16-supplement-3.

\section{Funding}

The study of diploid species was done under the framework of the State Budget Programme (project № 0324-2015-0005), the analysis of tetraploid wheats as well as the publication costs were funded by the Russian Scientific Foundation (Project No. 14-14-00161).

\section{Availability of data and materials}

All DNA sequences obtained in this study were deposited in the National Center for Biotechnology Information (NCBI) and received accession no. KX344100-18.

\section{Authors' contributions}

ABS designed the study, carried out the molecular and bioinformatics experiments and greenhouse analyses, and prepared the manuscript. AAS contributed to the molecular analysis. EAS helped with interpretation of the results and critically revised the manuscript. All authors read and approved the final manuscript

\section{Competing interests}

The authors declare that they have no competing interests.

\section{Consent for publication}

Not applicable.

\section{Ethics approval and consent to participate}

Not applicable.

\section{Author details}

${ }^{1}$ The Federal Research Center "Institute of Cytology and Genetics of Siberian Branch of the Russian Academy of Sciences", Lavrentiev ave. 10, Novosibirsk 630090, Russia. ${ }^{2}$ Institute of General Genetics of the Russian Academy of Sciences, Gubkina str., 3, Moscow 119991, Russia.

\section{Published: 16 November 2016}

\section{References}

1. Huang S, Sirikhachornkit A, Su X, Faris J, Gill B, Haselkorn R, Gornicki P. Genes encoding plastid acetyl-CoA carboxylase and 3-phosphoglycerate kinase of the Triticum/Aegilops complex and the evolutionary history of polyploid wheat. Proc Natl Acad Sci U S A. 2002;99:8133-8.

2. Marcussen T, Sandve SR, Heier L, Spannagl M, Pfeifer M, et al. Ancient hybridizations among the ancestral genomes of bread wheat. Science. 2014; 345(6194):1250092.

3. Badaeva ED, Boguslavsky RL, Badaev NS, Zelenin AV. Intraspecific chromosomal polymorphism of Triticum araraticum (Poaceae) detected by C-banding technique. P1 Syst Evol. 1990;169:13-24.
4. Goncharov NP. Comparative genetics of wheats and their related species. Novosibirsk: Siberian University Press (In Russian); 2002. p. 251.

5. Dvorak J, Zhang HB. Variation in repeated nucleotide sequences sheds light on the phylogeny of the wheat B and G genomes. Proc Natl Acad Sci U S A. 1990:87:9640-4

6. Mori N, Liu YG, Tsunewaki K. Wheat phylogeny determined by RFLP analysis of Nuclear-DNA. 2. Wild tetraploid wheats. Theor Appl Genet. 1995;90:129-34.

7. Kilian B, Ozkan H, Deusch O, Effgen S, et al. Independent wheat B and G genome origins in outcrossing Aegilops progenitor haplotypes. Mol Biol Evol. 2007;24:217-27.

8. Mitrofanova O, Badaeva E, Salina EA. T. timopheevii and T. zhukovskii, the bread wheat cousins and their contribution to T. aestivum improvement. In: Bonjean AP, Angus WJ, VanGinkel M, editors. The World Wheat Book: A History of Wheat Breeding (v.3). France: Tec \& Doc Lavoisier; 2016. p. 1167228. part 4, chapter 39.

9. Badaeva ED, Badaev NS, Gill BS, Filatenko AA. Intraspecific karyotype divergence in Triticum araraticum (Poaceae). P1 Syst Evol. 1994;192:117-45.

10. Yan L, Loukoianov A, Tranquilli G, Helguera M, et al. Positional cloning of the wheat vernalization gene VRN1. Proc Natl Acad Sci U S A. 2003;100:6263-8.

11. Trevaskis B, Hemming MN, Dennis ES, Peacock WJ. The molecular basis of vernalization induced flowering in cereals. Trends Plant Sci. 2007:12:352-7.

12. Distelfeld A, Li C, Dubcovsky J. Regulation of flowering in temperate cereals. Curr Opin Plant Biol. 2009:12:1-7.

13. Yan L, Helguera M, Kato K, Fukuyama S, Sherman J, Dubcovsky J. Allelic variation at the VRN-1 promoter in polyploid wheat. Theor Appl Genet. 2004;109:1677-86.

14. Fu D, Szucs P, Yan L, Helguera M, Skinner JS, vonZitzewitz J, et al. Large deletions within the first intron in VRN-1 are associated with spring growth habit in barley and wheat. Mol Gen Genomics. 2005;273:54-65.

15. Shcherban AB, Strygina KV, Salina EA. VRN-1 gene- associated prerequisites of spring growth habit in wild tetraploid wheat $T$. dicoccoides and the diploid $A$ genome species. BMC Plant Biol. 2015;15:94. doi:10.1186/s12870-015-0473-X.

16. Magness JR, Markle GM, Compton CC: Food and feed crops of the United States. Interregional Research Project IR-4, 1971, IR Bul. 1 (Bul. 828 New Jersey Agr. Expt. Sta.). https://hort.purdue.edu/newcrop/CroplnfoSources/ Magness info.htm.

17. Ivanicova Z, Jakobson I, Reis D, Safar J, Milec Z, et al. Characterization of new allele influencing flowering time in bread wheat introgressed from Triticum militinae. New Biotech. 2016;33(5):718-27.

18. Li C, Dubcovsky J. Wheat FT protein regulates VRN1 transcription through interactions with FDL2. Plant J. 2008:55(4):543-54.

19. Chen A, Li C, Hu W, Lau MY, et al. PHYTOCHROME C plays a major role in the acceleration of wheat flowering under long-day photoperiod. Proc Natl Acad Sci U S A. 2014;111(28):10037-44.

20. Loukoianov A, Yan L, Blechi A, Sanchez A, Dubcovsky J. Regulation of VRN-1 vernalization genes in normal and transgenic polyploid wheat. Plant Physiol. 2005:138:2364-73.

21. Dubcovsky J, Loukoianov A, Fu D, Valarik M, et al. Effect of photoperiod on the regulation of wheat vernalization genes V rn-1 and Vrn-2. Plant Mol Biol. 2006;60:469-80

22. Oliver SN, Deng W, Casao MC, Trevaskis B. Low temperatures induce rapid changes in chromatin state and transcript levels of the cereal VERNALIZATION1 gene. J Exp Bot. 2013:64:2413-22.

23. Shcherban AB, Efremova TT, Salina EA. Identification of a new Vrn-B1 allele using two near-isogenic wheat lines with difference in heading time. Mol Breed. 2012;29(3):675-85.

24. Shcherban $A B$, Khlestkina EK, Efremova TT, Salina EA: The effect of two differentially expressed wheat VRN-B1 alleles on the heading time is associated with structural variation in the first intron. Genetica 2013, 141:133-141.

25. Goncharov NP. Genetic resources of wheat related species: The Vrn genes controlling growth habit (spring vs. winter). Euphytica. 1998;100:371-6.

26. Levy AA, Feldman M. The impact of polyploidy on grass genome evolution Plant Physiol. 2002;130:1587-93.

27. Golovnina KA, Kondratenko E, Blinov AG, Goncharov NP. Molecular characterization of vernalization loci VRN1 in wild and cultivated wheats. BMC Plant Biol. 2010;10:168.

28. Feldman M. The origin of cultivated wheat. The World Wheat Book. Paris: Lavoisier Publishing: 2001. p. 3-58.

29. von Zitzewitz J, Szucs P, Dubcovsky J, Yan L, Francia E, et al. Molecular and structural characterization of barley vernalization genes. Plant Mol Biol. 2005, 59:449-67. 
30. Oliver SN, Finnegan EJ, Dennis ES, Peacock WJ, Trevaskis B. Vernalizationinduced flowering in cereals is associated with changes in histone methylation at the VERNALIZATION1 gene. Proc Natl Acad Sci U S A. 2009;106:8386-91.

31. Salina EA, Lim YK, Badaeva ED, Shcherban AB, et al. Philogenetic reconstruction of Aegilops section Sitopsis and the evolution of tandem repeats in the diploids and derived wheat polyploids. Genome. 2006:49:1023-35.

32. Pidal B, Yan L, Fu D, Zhang F, Tranquilli G, Dubcovsky J. The CArG-box located upstream from the transcription start of wheat vernalization gene VRN-1 is not necessary for the vernalization response. J Heredity. 2009;100: $355-64$

33. Muterko A, Kalendar R, Salina E. Novel alleles of the VERNALIZATION1 genes in wheat are associated with modulation of DNA curvature and flexibility in the promoter region. BMC Plant Biol. 2016;16(S1):65-81.

34. Gill BS, Li W, Sood S, Kuraparthy V, Friebe BR, et al. Genetics and genomics of wheat domestication-driven evolution. IJPS. 2007:55:223-9.

35. Kiseleva AA, Shcherban AB, Leonova IN, Frenkel Z, Salina EA: Identification of new heading date determinants in wheat $5 \mathrm{~B}$ chromosome. BMC Plant Biol. 2016, DOI 10.1186/s12870-015-0688-x

36. Thompson JD, Higgins DG, Gibson TJ. CLUSTAL W: improving the sensitivity of progressive multiple sequence alignment through sequence weighting, position-specific gap penalties and weight matrix choice. Nucleic Acids Res. 1994:22:4673-80.

37. Tamura K, Dudley J, Nei M, Kumar S. MEGA4: Molecular Evolutionary Genetics Analysis (MEGA) software version 4.0. Mol Biol Evol. 2007;24:1596-9.

38. Blake NK, Lehfeldt BR, Lavin M, Talbert LE. Phylogenetic reconstruction based on low copy DNA sequence data in an allopolyploid: the B genome of wheat. Genome. 1999;42:351-60.

\section{Submit your next manuscript to BioMed Central and we will help you at every step:}

- We accept pre-submission inquiries

- Our selector tool helps you to find the most relevant journal

- We provide round the clock customer support

- Convenient online submission

- Thorough peer review

- Inclusion in PubMed and all major indexing services

- Maximum visibility for your research

Submit your manuscript at www.biomedcentral.com/submit 